\title{
Tobacco use and measuring nicotine dependence among urban Indigenous pregnant women
}

\author{
Kathryn S Panaretto, Melvina R Mitchell, Lynette Anderson, Conor Gilligan, Petra Buettner, Sarah L Larkins and Sandra Eades
}

S moking cessation interventions, involving pharmacological, behavioural and alternative therapies have been extensively studied overseas and in mainstream communities in Australia. While evidence from these studies can influence the design of quit-smoking interventions for Aboriginal people and Torres Strait Islanders, it has some limitations. ${ }^{1}$ Many of the intervention studies enrol predominantly moderately to highly nicotinedependent smokers and are conducted in communities where smoking is no longer the norm. ${ }^{2,3}$ Consequently, pharmacological support has assumed an increasingly dominant role in mainstream quit programs.

One of the factors in determining whether an individual stops smoking is physical nicotine addiction. There are some data from the Indigenous community that describe motivation to quit and the contextual factors in the environment that will affect the habitual and psychological aspects of smoking, but no data on physical addiction to nicotine. ${ }^{1,4}$ The Fagerström Test for Nicotine Dependence (FTND) is a validated tool that uses six short questions to assess a person's physical nicotine dependence. ${ }^{5}$ Its brevity and reliability in predicting relapse ${ }^{6}$ has led to it being widely used in the evaluation of smoking cessation interventions, and its use is recommended in Australian protocols. ${ }^{7}$ However, there is little evidence from within the Indigenous community to inform its use.

We aimed to further investigate the questions of nicotine dependence and the usefulness of the FTND in the Indigenous population. In conjunction with a randomised trial of an intervention to reduce smoking in pregnant Aboriginal and Torres Strait Islander women (the Tilly's Tracks project), the staff of the Townsville Aboriginal and Islander Health Service (TAIHS) Mums and Babies program examined patterns of nicotine dependence, the value of the FTND, and its correlation with selfreported tobacco use and urinary cotinine concentrations among pregnant women attending TAIHS for antenatal care.

\section{METHODS}

The Mums and Babies program, involving antenatal care delivered by a mutidisciplin-

\section{ABSTRACT}

Objectives: To examine patterns of nicotine dependence, the value of the Fagerström Test for Nicotine Dependence (FTND) and its correlation with self-reported tobacco use and urinary cotinine concentrations among pregnant Indigenous women in Townsville.

Design, participants and setting: Cross-sectional study of 201 consecutive women who self-reported tobacco use at their first antenatal visit to Townsville Aboriginal and Islander Health Service (TAIHS) between 1 November 2005 and 31 October 2007. All smokers were to be assessed by FTND, and 108 women participating in the Tilly's Tracks project (a randomised trial of an intervention to reduce smoking in pregnant Aboriginal and Torres Strait Islander women) were to have a comprehensive smoking history taken and urinary cotinine samples collected.

Main outcome measures: Self-reported smoking status, FTND scores and urinary cotinine concentrations.

Results: Of 302 Indigenous women presenting to TAIHS, 201 (66.6\%) identified as current tobacco users at their first antenatal visit; this proportion rose to $79.6 \%$ in women aged $<20$ years. An FTND was completed for 152 women (75.6\%), with a median score of 4 , and $40.1 \%$ scoring 3 or less, indicating low levels of nicotine dependence. There were significant correlations between the FTND and number of cigarettes smoked $(\rho=0.56 ; P<0.001)$ and urinary cotinine concentrations $(\rho=0.25 ; P=0.030)$. Of those who provided comprehensive smoking histories, the median age of starting smoking was 15 years, with a median of two previous quit attempts; $71.4 \%$ reported partners who smoked and $27.3 \%$ reported smoking occurred inside the house.

Conclusion: The use of the FTND in Indigenous pregnant women may assess physical nicotine dependence, thus providing information that will help in preparing quitsmoking plans, including tailoring of pharmacological support to individual need. Quit-smoking programs that better address the behavioural and psychological aspects of smoking within the Indigenous community in Australia are needed.

MJA 2009; 191: 554-557

ary team, which commenced on 1 January 2000 in Townsville, Queensland, has been described previously. ${ }^{8}$ From early 2005, all women attending the Mums and Babies program who reported tobacco use have been assessed using the FTND to guide brief interventions for tobacco use. In addition, from 1 November 2005, women undergoing baseline assessment for the Tilly's Tracks project $^{9}$ provided a comprehensive smoking history, including a 24-hour retrospective diary detailing their personal smoking status, their partner's smoking habits, and exposure to environmental tobacco smoke in the home. This information was collected at interview by trained Aboriginal health workers, and was added to the routine antenatal care activities included in a first visit by the clinical team. Women also provided urine samples for cotinine testing.

In this study, we examined antenatal firstvisit data for all Indigenous women who self-reported as smokers, and attended TAIHS for antenatal care during the 2 years between 1 November 2005 and 31 October 2007. In addition, we added a qualitative component involving a focus group discussion with Mums and Babies program staff to evaluate the usefulness and ease of use of the FTND in the context of day-to-day activity in a busy primary care clinic.

Staff asked all women whether they identified as Aboriginal, Torres Strait Islander, both Aboriginal and Torres Strait Islander, or other identities.

Cotinine analysis was performed on urine samples of the women who were involved in the Tilly's Tracks project. Analysis was by enzyme-linked immunosorbent assay (ELISA) (Cozart Microplate EIA, Cozart, Abingdon, Oxfordshire, UK), as described previously. ${ }^{9}$ A standard curve was generated for each analysis run, and if the cotinine levels for both samples in a duplicate pair were above the standard 


\section{Derivation of the 302 pregnant Indigenous women included in the study}

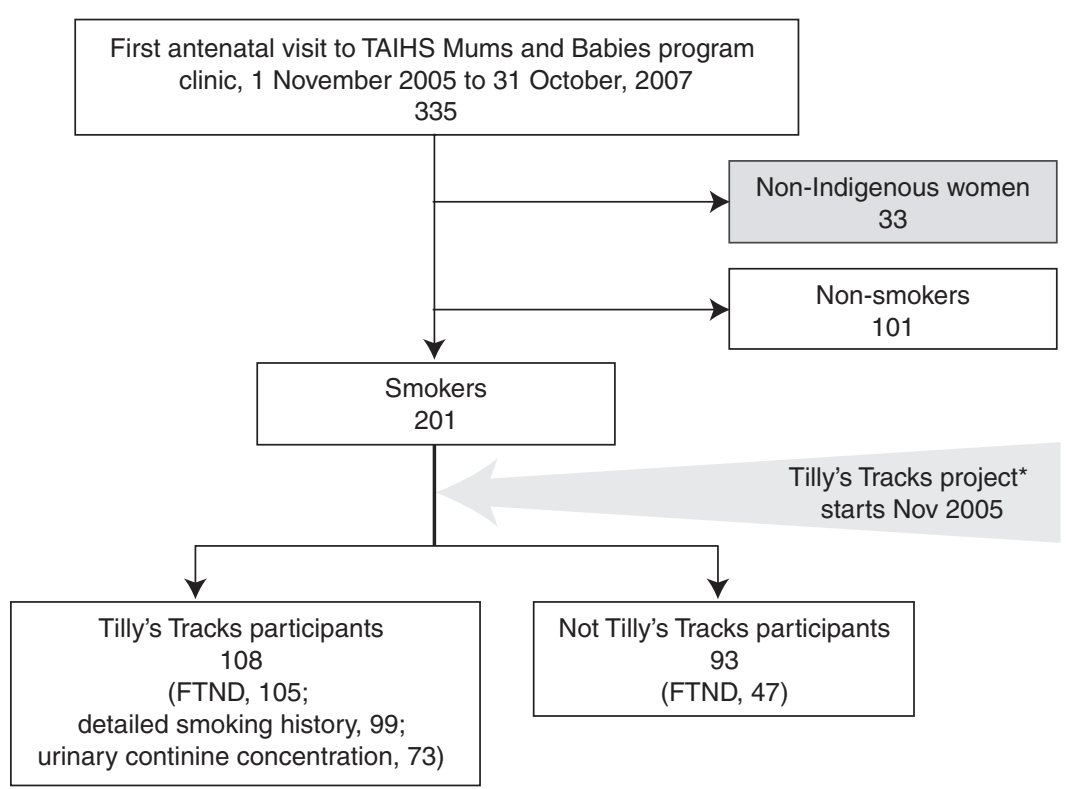

TAIHS = Townsville Aboriginal and Islander Health Service. FTND = Fagerström Test for Nicotine Dependence. * The Tilly's Tracks project included pregnant Indigenous women who: were smokers; agreed to participate; attended for their first antenatal care visit before 20 weeks' gestation; were Townsville residents; did not have mental illness; and were not being treated for the use of any substance other than tobacco.

\section{Comparison of demographic and other characteristics for smokers and non- smokers among 302 pregnant Australian Indigenous women attending the Mums and Babies program at the Townsville Aboriginal and Islander Health Service between 1 November 2005 and 31 October 2007}

\begin{tabular}{lcccc} 
Characteristic & $\begin{array}{c}\text { Total } \\
(n=302)\end{array}$ & $\begin{array}{c}\text { Non-smoker } \\
(n=101)\end{array}$ & $\begin{array}{c}\text { Smoker } \\
(n=201)\end{array}$ & $P$ \\
\hline Age $<20$ years & $17.6 \%$ & $10.6 \%$ & $21.2 \%$ & 0.026 \\
Single/separated/divorced & $37.9 \%$ & $36.0 \%$ & $38.8 \%$ & 0.636 \\
Education to Year 10 or below & $44.3 \%$ & $29.3 \%$ & $51.9 \%$ & 0.001 \\
Tobacco use & $100 \%$ & $33.4 \%$ & $66.6 \%{ }^{*}$ & \\
Alcohol use & & & & \\
$\quad$ Any & $26.2 \%$ & $9.9 \%$ & $34.3 \%$ & 0.001 \\
$\quad$ Risky & $16.2 \%$ & $4 \%$ & $22.4 \%$ & 0.001 \\
Recreational drug use & $13.9 \%$ & $6.9 \%$ & $17.4 \%$ & 0.013 \\
Domestic violence & $9.9 \%$ & $8.9 \%$ & $10.4 \%$ & 0.674 \\
\hline
\end{tabular}

* Median, 10 cigarettes/day; interquartile range, 5-15 cigarettes/day. † Risky drinker according to national clinical guidelines, $2006 .{ }^{10} P<0.05$ significant.

range, that sample was allocated the maximum level from the standard curve for the run.

A comprehensive record of each pregnancy at TAIHS is recorded in a secure database. The FTND data are included in this data collection. All other Tilly's Tracks project data are held in a secure database at the University of Newcastle.

\section{Statistical analysis}

Depending on their distribution, numerical data are reported as mean and SD or median and interquartile range (IQR). Bivariate associations were tested using $\chi^{2}$, KruskalWallis and $t$ tests, as appropriate. Spearman rank correlation coefficients were calculated to assess the correlation between the FTND score and (i) numbers of cigarettes smoked and (ii) the measured urinary cotinine concentration as assessed at the first visit.

All statistical tests were performed using SPSS Version 17.0 for Windows (SPSS Inc, Chicago, Ill, USA). A two-tailed $P$ value below 0.05 was regarded as significant.

\section{Ethics approval}

The smoking cessation project had the full support of the community elected Board of TAIHS. The Human Research Ethics Committee of the University of Newcastle approved the research project. All women gave written consent to participate.

\section{RESULTS}

Box 1 shows the derivation of the study groups. Of the 302 pregnant Indigenous women, $71.9 \%$ identified as Aboriginal, $17.2 \%$ as Torres Strait Islanders and $10.9 \%$ as both, and 201 (66.6\%) self-reported current tobacco use at their first antenatal care visit.

The mean age of the 302 women was 25.6 years (SD, 5.9), with no difference between smokers and non-smokers. Of the women aged less than 20 years, $79.6 \%$ reported current tobacco use (Box 2). The only characteristic that differed between women who did or did not complete an FTND was alcohol use, with those not completing the FTND being more likely to report risky levels of drinking. There were no significant differences in demographic characteristics between the women in the Tilly's Tracks project who did and did not provide a urinary sample for cotinine estimation.

\section{Patterns of nicotine dependence}

Of the 201 smokers, 152 (75.6\%) completed an FTND. The distribution of FTND responses and scores is shown in Box 3. The median FTND score was 4 (IQR, 2-9); 40.1\% of these pregnant smokers scored 3 or less on the FTND. The FTND questions that correlated best with the total FTND score were Question 1 (How soon after you get out of bed do you have a cigarette?) and Question 4 (the number of cigarettes smoked each day). There was a significant positive correlation between FTND score and number of cigarettes smoked each day, with the number of cigarettes smoked explaining 30.3\% of the variation in the FTND (Box 4, A).

\section{Comprehensive smoking histories and cotinine levels}

A comprehensive smoking history was obtained for 99 women. The median age at which they started smoking was 15 years (IQR, 13-17; range, 9-32 years) and these women had made a median of two previous quit attempts (IQR, 1-3; range, 1-25). Of the 77 participants with partners, $71.4 \%$ had partners who smoked, $6.5 \%$ had partners who were former smokers and $22.1 \%$ had partners who had never smoked. Participants cohabited with a median of two 


\section{Patterns and distribution of nicotine dependence as assessed using the Fagerström Test of Nicotine Dependence (FTND) at the first antenatal visit for 152 women attending the Mums and Babies program clinic at Townsville Aboriginal and Islander Health Service between November 12005 and October 312007}

A. Patterns of nicotine dependence

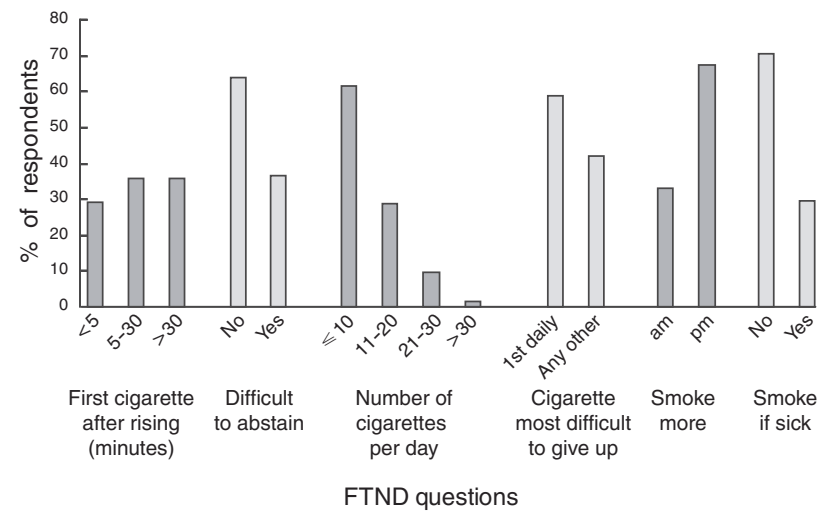

B. Distribution of nicotine dependence

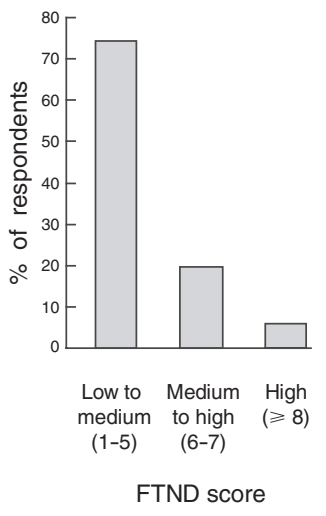

smokers (IQR, 1-3; range, 1-9), with $61.9 \%$ of households having three or more smokers in residence (including the trial participant). Smoking occurring mostly outside the house was reported by $64.6 \%$ of participants, while $27.3 \%$ reported that smoking occurred both inside and outside and $8.1 \%$ reported smoking mostly occurring inside the house; $37.4 \%$ of participants reported that smoking occurred inside the car.
A first-visit urinary cotinine sample was collected from 73 of the 108 women in the Tilly's Tracks project (67.6\%), with a median concentration of $3056.6 \mathrm{ng} / \mathrm{mL}$ (IQR, 1068.9$5858.7 \mathrm{ng} / \mathrm{mL})$. There was a significant positive correlation between FTND score and urinary cotinine concentrations (Box 4, B).

\section{Staff feedback}

The experience of the TAIHS staff, particularly that of Aboriginal Health Workers, indi-
4 Correlations between Fagerström Test for Nicotine Dependence (FTND) score and $(A)$ the reported number of cigarettes smoked (152 women), and (B) the urinary cotinine concentration measured ( 73 women) at the first antenatal visit to the Mums and Babies program clinic at the Townsville Aboriginal and Islander Health Service between 1 November 2005 and 31 October 2007
A. Correlation between FTND score and number of cigarettes smoked (152 women)

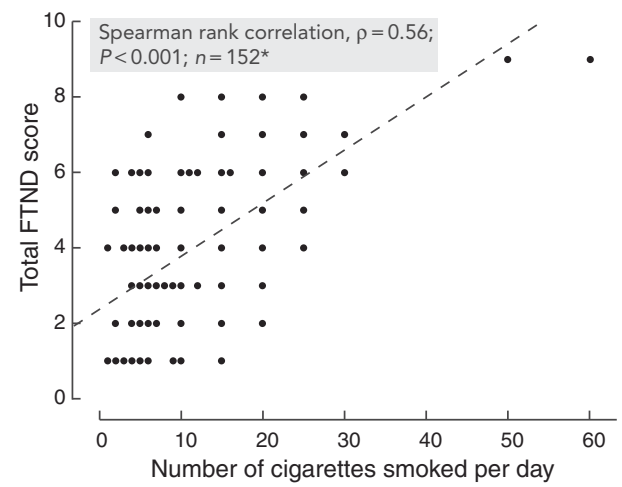

B. Correlation between FTND score and urinary cotinine concentration (73 women)

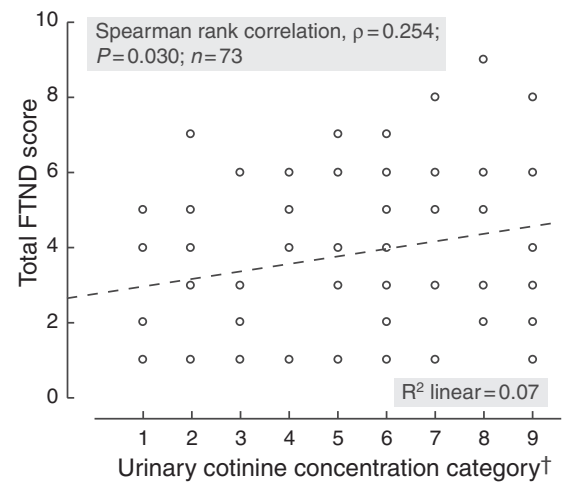

* If the data are reanalysed without the two outliers (reporting smoking 50 and 60 cigarettes/day), the Spearman rank correlation is $\rho=0.55 ; P<0.001$. † Cotinine concentration $(\mathrm{ng} / \mathrm{mL})$ categories were based on the percentiles of the original distribution of cotinine: $1, \leqslant 300 ; 2,301-800 ; 3,801-1500 ; 4,1501-2100 ; 5$, 2101-3000; 6, 3001-5000; 7, 5001-5750; 8, 5751-6000; 9, > 6000 . cated that the FTND was easy and reasonably quick to use, and readily incorporated into daily workloads. Only Question 2 required explanation (the term "abstain" was unfamiliar to some patients). Staff members felt that the FTND gave them a good entry point to a discussion about smoking in the context of broaching such a discussion with women from households with a certain amount of social chaos, multiple resident smokers and competing health priorities; staff also felt women were honest in their answers. Staff members also commented that they found the scores provided a visual element to counselling about the level of dependence on nicotine and helped them to encourage quit attempts during their brief intervention work.

\section{DISCUSSION}

Using the FTND, a well validated tool to measure nicotine dependence, most pregnant Indigenous women in this study scored in the lowest category, indicating a low to medium physical dependence on nicotine.

Most smoking cessation guidelines suggest that an assessment of nicotine dependence should be made to guide individual quit attempts. ${ }^{7,11,12}$ Use of the FTND in this study was incorporated into the workflow of a busy Indigenous maternal and child health clinic. Feedback from health workers and other staff was positive. They found the tool simple to incorporate into daily clinical work, easily understood and useful as an "ice breaker". The study shows significant correlations between the number of cigarettes smoked and nicotine dependence scores. The correlations of both the number of cigarettes smoked and the time until the first cigarette of the day - the two FTND questions that comprise the Heavy Smoking Index $(\mathrm{HSI})^{11}$ — with the FTND score are to be expected, but many women had low total FTND scores, and the other FTND questions could potentially have been significant contributors to the total FTND score. With international evidence suggesting that relying solely on the number of cigarettes smoked as an indicator of nicotine dependence will underestimate the number of patients with a high level of nicotine addiction, ${ }^{13}$ the FTND or HSI may be useful in primary care clinics.

The level of nicotine dependence in the Indigenous community in Australia has not been previously reported. Among the group of Indigenous women in our study, 40\% scored 3 or lower on the FTND, indicating low levels of physical nicotine dependence. 
This distribution of scores is consistent with findings of other studies in pregnant women. ${ }^{14,15}$ It is possible that our cohort of Indigenous women under-scored on the FTND, as has been suggested in African American teenagers. ${ }^{16}$ However, the credibility of these demonstrated lower levels of nicotine dependence may be supported by the current Indigenous tobacco narrative, which includes suggestions that Indigenous people cope without constant access to cigarettes, ${ }^{1}$ and an apparent failure of nicotine replacement therapy (NRT) in the Northern Territory. ${ }^{17}$ The NT trial showed that NRT conferred little advantage over a brief intervention alone, which was partly blamed on inadequate resources and a low-intensity intervention. However, the degree of nicotine dependence was not reported in that study, and it is possible that the nicotine dependence of the participants may have been lower than was assumed, ${ }^{4}$ which might have contributed to the lack of efficacy of NRT.

Low levels of nicotine dependence among Indigenous pregnant women has significant implications for the implementation of quitsmoking programs. Evidence suggests that NRT is not of value in light smokers or those with low nicotine dependence, ${ }^{18,19}$ which is reflected in a number of smoking cessation guidelines. $^{7,12}$ This reinforces the need to assess nicotine dependence. While pharmacological therapies are important, they are expensive, have side effects, and their use must be tailored to individual needs. The FTND scores in this study suggest cessation programs need to consider the smoking behaviours of each Indigenous woman to develop individual quit plans with strategies to counter the habitual aspects of their smoking.

Our study had some limitations. The cohort comprised pregnant women, and the overall demographic characteristics of our cohort were typical of Indigenous pregnant women, ${ }^{8}$ so our sample was probably representative of that group. However, our findings may not be applicable to the nonpregnant Indigenous community. The FTND has previously been found to be psychometrically sound and to correlate strongly with cotinine levels;, 20 the significant but weaker association found in this group of women may be explained by a number of factors. Nicotine metabolism in pregnancy is accelerated, resulting in reduced cotinine levels, ${ }^{21}$ and cigarette consumption tends to vary throughout pregnancy. ${ }^{22}$ In addition, recording cotinine levels using a maximum cut-off level is likely to have underestimated the correlation coefficient for FTND and cotinine level. While cotinine levels are method-dependent, ${ }^{20,23}$ those reported for these pregnant women are comparable to levels found in other reports. ${ }^{22,24}$ To further explore these issues in the Indigenous Australian community would require more rigorous investigation.

In conclusion, this study shows the use of the FTND in Indigenous pregnant women may assess physical nicotine dependence, thus providing information that will help in preparing quit-smoking plans. Tailored use of pharmacological support must be assisted by strategies to address habitual and psychological aspects of smoking within the Indigenous community in Australia.

\section{ACKNOWLEDGEMENTS}

We thank the Board of TAlHS for their support of the project. We also thank Kaye Thompson for her work in the Tilly's Tracks project. This study was supported in part by National Health and Medical Research Council Project Grant No. 510776.

\section{COMPETING INTERESTS}

None identified.

\section{AUTHOR DETAILS}

Kathryn S Panaretto, MBBS, FAFPHM, Population Health Medical Officer ${ }^{1}$

Melvina R Mitchell, EN, Senior Health Worker ${ }^{2}$ Lynette Anderson, EN, Chronic Disease Coordinator $^{1}$

Conor Gilligan, BPsych, PhD, Lecturer, Discipline of Health Behaviour Sciences ${ }^{3}$

Petra Buettner, PhD, Senior Lecturer, Skin Cancer Research Group, School of Public Health, Tropical Medicine and Rehabilitation Sciences ${ }^{4}$

Sarah L Larkins, MBBS, MPH\&TM, PhD, Senior Lecturer, Primary Health Care, and Course Coordinator, Graduate Certificate in Primary Health Care Research (PHCRED Program), Rural Health Research Unit, School of Medicine and Dentistry ${ }^{4}$ Sandra Eades, MBBS, PhD, Research Fellow ${ }^{5}$ 1 Queensland Aboriginal and Islander Health Council, Queensland Aboriginal and Islander Health Population Health Hub, Townsville, QLD.

2 Community Child Health Unit, Queensland Health, Townsville, QLD.

3 School of Medicine and Public Health, University of Newcastle, Newcastle, NSW.

4 James Cook University, Townsville, QLD.

5 Baker Institute, Melbourne, VIC.

Correspondence: katiepanaretto@qaihc.com.au

\section{REFERENCES}

1 Ivers RG. An evidence-based approach to planning tobacco interventions for Aboriginal people. Drug Alcohol Rev 2004; 23: 5-9.

2 Baker A, Ivers RG, Bowman J, et al. Where there's smoke, there's fire: high prevalence of smoking among some subpopulations and recommendations for intervention. Drug Alcohol Rev 2006; 25: 85-96.
3 Shiffman S. Reflections on smoking relapse research. Drug Alcohol Rev 2006; 25: 15-20.

4 Johnston V, Thomas D. Smoking behaviours in a remote Australian Indigenous community: the influence of family and other factors. Soc Sci Med 2008; 67: 1708-1716.

5 Heatherton TF, Kozlowski LT, Frecker RC, Fagerström KO. The Fagerström Test for Nicotine Dependence: a revision of the Fagerström Tolerance Questionnaire. Br J Addict 1991; 86: 1119-1127

6 Piper M, McCarthy D, Baker T. Assessing tobacco dependence: a guide to measure evaluation and selection. Nicotine Tob Res 2006; 8: 339-351.

7 Royal Australian College of General Practitioners. Smoking cessation guidelines for Australian general practice: Practice handbook 2004. Melbourne: RACGP, 2005.

8 Panaretto KS, Lee HM, Mitchell MR, et al. Impact of a collaborative shared antenatal care program for urban Indigenous women: prospective cohort study. Med J Aust 2005; 182: 514-519.

9 Gilligan C, Sanson-Fisher R, Eades S, et al. Assessing the accuracy of self-reported smoking status among pregnant Aboriginal and Torres Strait Islander women using cotinine biochemical validation. Drug Alcohol Rev 2009. In press.

10 Ministerial Council on Drugs Strategy. National Drug Strategy. Aboriginal and Torres Strait Islander peoples complementary action plan 2003-2006. Background paper. Canberra: National Drug Strategy, 2003. (accessed Oct 2009).

11 Royal Australian College of General Practitioners. SNAP: a population health guide to behavioural risk factors in general practice. Melbourne: RACGP, 2004.

12 van Schayck OC, Pinnock H, Ostrem A, et al. International Primary Care Respiratory Group consensus statement. Tackling the smoking epidemic - practical guidance for primary care. Prim Care Respir J 2008; 17: 185-193.

13 de Leon J, Diaz FJ, Becoña E, et al. Exploring brief measures of nicotine dependence for epidemiological surveys. Addict Behav 2003; 28: 1481-1486.

14 Ward K, Vander Weg M, Sell M, et al. Characteristics and correlates of quitting smoking among black and white lowincome pregnant smokers. Am J Health Behav 2006; 30: 651-662.

15 Händel G, Hannöver W, Röske K, et al. Intention to change smoking in pregnant and postpartum women according to number of pregnancies. Eur J Public Health 2009; 19: 218221.

16 Schroeder JR, Moolchan ET. Ethnic differences among adolescents seeking smoking cessation treatment: a structural analysis of responses on the Fagerström Test for Nicotine Dependence. Nicotine Tob Res 2007; 9: 137-145.

17 Ivers RG, Farrington M, Burns CB, et al. A study of the use of free nicotine patches by Indigenous people. Aust NZ J Public Health 2003; 27: 486-490.

18 Tang J, Law M, Wald N. How effective is nicotine replacement therapy in helping people to stop smoking? BMJ 1994; 308: 21-26.

19 Peters MJ, Morgan LC. The pharmacotherapy of smoking cessation. Med J Aust 2002; 176: 486-490.

20 Haufroid V, Lison D. Urinary cotinine as a tobacco-smoke exposure index: a minireview. Int Arch Occup Environ Health 1998; 71: 162-168.

21 Dempsey D, Jacob Pr, Benowitz N. Accelerated metabolism of nicotine and cotinine in pregnant smokers. J Pharmacol Exp Ther 2002; 301: 594-598.

22 Pickett K, Rathouz P, Kasza K, et al. Self-reported smoking, cotinine levels, and patterns of smoking in pregnancy. Paediatr Perinat Epidemiol 2005; 19: 368-376.

23 Gorber S, Schofield-Hurwitz S, Hardt J, et al. The accuracy of self-reported smoking: a systematic review of the relationship between self-reported and cotinine-assessed smoking status. Nicotine Tob Res 2009; 11: 12-24.

24 Lawrence T, Aveyard P, Croghan E. What happens to women's self-reported cigarette consumption and urinary cotinine levels in pregnancy? Addiction 2003; 98: 1315-1320.

(Received 2 Apr 2009, accepted 30 Aug 2009) 